\title{
The Effects of METTL3-Mediated Regulations of the Adipogenic Differentiations of Bone Marrow MSCs Targeting PI3K/AKT Pathways on the Chemo- Resistance of AML
}

\section{Zhipeng Pan}

Fujian Medical University Union Hospital

\section{Bin Wang}

Fujian Medical University Union Hospital

Diyu Hou

Fujian Medical University Union Hospital

Ruolan You

Fujian Medical University Union Hospital

\section{Xiaoting Wang}

Fujian Medical University Union Hospital

\section{Wenhui Xie}

Fujian Medical University Union Hospital

Huifang Huang ( $\square$ huanghuif@fjmu.edu.cn )

Xiehe Affiliated Hospital of Fujian Medical University https://orcid.org/0000-0002-8548-028X

\section{Research}

Keywords: AML, MSCs, adipogenic differentiations, m6A, chemo-resistance

Posted Date: April 15th, 2020

DOI: https://doi.org/10.21203/rs.3.rs-22094/v1

License: (c) (1) This work is licensed under a Creative Commons Attribution 4.0 International License. Read Full License 


\section{Abstract}

Background: The adipogenic differentiations of bone marrow mesenchymal stem cells (MSCs) result in the promotion of the chemo-resistance of AML cells. When compared with bone marrow MSCs from healthy donors (HD-MSCs), it has been observed that the MSCs from AML (AML-MSCs) displayed enhanced adipogenic differentiation abilities. However, the underlying mechanism remains elusive. METTL3-mediated modifications of $\mathrm{m}^{6} \mathrm{~A}$ mRNA play key roles in the regulation of stem cell differentiation. Unfortunately, there has been no research to date which has reported the fact that the increases of adipogenic differentiation of AML-MSCs are regulated by the $\mathrm{m}^{6} \mathrm{~A}$. The goal of this study was to investigate the critical role of METTL3 in the adipogenesis of AML-MSCs for promoting the chemo-resistance of AML.

Methods: This study detected the m6A levels of the MSCs total RNA, and then investigated METTL3 functions in the adipogenic differentiations of MSCs using knockdown and overexpression experimental methods. The effects of the adipogenic differentiations of HD-MSCs/AML-MSCs on the chemo-resistance to $A M L$ were also examined. In addition, the gene transcriptome and $\mathrm{m}^{6} \mathrm{~A}$ epigenetic changes of $\mathrm{AML}-$ MSCs from four AML patients, and the HD-MSCs from three healthy donors, were analyzed in this study. The differentially expressed genes and pathways were successfully identified, and clinical specimens were used to adjust gene expressions in order to determine their effects on the adipogenic differentiations of MSC.

Results: The results demonstrated that METTL3 could significantly inhibit the adipogenic differentiations of MSCs. It was found that when compared with the HD-MSCs, the expressions of the PI3K/AKT signaling pathways in the AML-MSCs were significantly increased. Meanwhile, the levels of $\mathrm{m}^{6} \mathrm{~A}$ were significantly decreased. Therefore, it was indicated that by blocking the PI3K/AKT signaling pathways with AKT inhibitors, the adipogenic differentiations of the MSCs could be significantly inhibited. Moreover, the increased adipogenic differentiations induced by the METTL3 could significantly increase the chemo-resistance of the AML.

Conclusions: It was found that the METTL3 had enhanced the modification levels of $\mathrm{m}^{6} \mathrm{~A}$ in the PI3K/AKT signaling pathways, and then reduced its expression. Subsequently, the adipogenic differentiations were inhibited. Then, the bone marrow microenvironments, as well as chemo-resistance of the AML, were successfully changed.

\section{Introduction}

Acute myeloid leukemia (AML) is the most common acute leukemia diagnosed in adults, and is characterized with a high recurrence rate ${ }^{[1,2]}$. Bone marrow microenvironments (BMM) and AML cells remodel to promote the development of the disease ${ }^{[3-5]}$. MSCs, which are important elements of the BMMs, are the precursors of osteoblasts and adipocytes in bone marrow ${ }^{[3]}$. The balance between the osteogenesis and adipogenesis is strictly regulated in order to ensure normal bone marrow functions ${ }^{[6,7]}$. 
The results of previous studies have shown that the MSCs from AML patients (AML-MSCs) have significant impairments of osteogenic differentiations, as well as remarkable enhancements of adipogenic differentiations ${ }^{[8]}$. The enhanced adipogenesis of the AML-MSCs has been found to promote the development of leukemia ${ }^{[9]}$.

The epigenetic regulation of mRNA is an important factor affecting stem cell differentiation ${ }^{[10]}$. $N^{6}$ methyladenine $\left(\mathrm{m}^{6} \mathrm{~A}\right)$ is the most abundant, dynamic, and reversible RNA modification for eukaryotic mRNA ${ }^{[11,12]}$. The $m^{6} A$ is carried out with the participation of methyltransferase complexes (writers, such as METTL3, METTL14, and WTAP); demethylase (erasers, such as ALKBH5 and FTO); and $\mathrm{m}^{6} \mathrm{~A}$ reader proteins (readers, such as YTH family and IGF2BP family), which have the ability to dynamically and reversibly regulate RNA splicing, transport, translation, and stability ${ }^{[13-15] .}$

Both the methylase and demethylase of the m6A play important roles in AML. Recent studies have found that METTL3 is highly expressed in AML cells, which demonstrated the ability to induce the progression of leukemia in AML transplanted mice ${ }^{[16]}$. FTO can promote the malignant transformation and leukemogenesis mediated by oncogene by reducing the mRNA $\mathrm{m}^{6} \mathrm{~A}$ levels of ASB2 and RARA ${ }^{[17]}$. It has been found that R-2HG plays an anti-leukemia role by targeting the FTO by increasing $\mathrm{m}^{6} \mathrm{~A}$ levels and inducing cycle arrest and apoptosis of the AML cells. However, the specific roles of $\mathrm{m}^{6} \mathrm{~A}$ modifications in the adipogenic differentiations of AML-MSCs have not yet been reported. In this study, RNA sequencing and $\mathrm{m}^{6} \mathrm{~A}$ microarrays, combined with clinical specimen validations, were used to analyze the mRNA and signal pathway differentially expressed in AML-MSCs ${ }^{[18]}$. The obtained results will provide a theoretical basis for determined new targets for AML treatments from the perspective of $\mathrm{m}^{6} \mathrm{~A}$ modifications and bone marrow microenvironmental conditions, which have potential value in future clinical applications.

\section{Materials And Methods}

\section{Primary bone marrow MSCs cultures}

In the current study, bone marrow specimens of AML-MSCs were obtained from the hematology department of Fujian Medical University Union Hospital. The collection of the bone marrow specimens was approved by the hospital's ethics review board, and obtained using an informed written consent process. Mononuclear cells (MNC) were isolated using a Ficoll-Hypaque Density Centrifugation Method. Then, the MNC were seeded in low-glucose Dulbecco's Modified Eagle Medium (LG-DMEM, Hyclone), supplemented with $20 \%$ Fetal Bovine Serum (FBS, Gibco) and 1\% Penicillin-Streptomycin (Hyclone). The cells were plated in $100 \mathrm{~mm}^{2}$ culture dishes at a density of $10^{6} \mathrm{cells} / \mathrm{ml}$ and incubated at $37^{\circ} \mathrm{C}$ with $5 \%$ $\mathrm{CO}_{2}$. Then, following a culturing period of one week, the non-adherent cells were removed, adherent MSCs were passaged with a confluence of 80 to $90 \%$, and the cells were expanded to passage $(P)$ four. The bone marrow specimens of HD-MSCs were purchased from the Cyagen Biosciences Company. The cultured MSCs were identified as CD34/CD44+/CD45/CD73+/CD90+/CD105+, whose positive rates were 
all greater than $95 \%$. In addition, the MSCs differentiations into osteocytes and adipocytes were conducted and had demonstrated multidirectional differentiation potential.

\section{MSC differentiations}

The MSCs were cultured in advance with $2 \times 10^{5}$ cells/well in six-well plates with $0.1 \%$ gelatin. Then, when the cultured cell fusion degree reached between $60 \%$ and $70 \%$, the cells were carefully sucked out in order the complete the culture medium, and $2 \mathrm{ml}$ of osteogenic differentiation culture medium were added. The induced differentiation was stained on the 21 st day $[19,20]$.

During the process of the MSCs culturing with $2 \times 10^{5}$ cells/well in six-well plates, the solution was changed every three days until the cell fusion reaches $100 \%$ or over fusion occurred. Then, the cell complete culture medium was carefully sucked out, and $2 \mathrm{ml}$ of adipogenic differentiation medium $\mathrm{A}$ were added to the six-well plates. After a period of three days, Solution A was sucked out and $2 \mathrm{ml}$ of differentiation medium solution B were added. Then, after 24 hours, Solution B was replaced with Solution $A$. The aforementioned steps were alternately repeated 3 to 5 times ( 12 to 20 days). The cultures with Solution B were maintained for three days until the lipid drops became large and round enough for the subsequent experiments. During the maintenance of Solution B, fresh Solution B was used every three days. Then, the specimens were stained using oil red $O{ }^{[20,21]}$.

\section{Alizarin red staining and oil red 0 staining methods}

For the alizarin red staining (ARS) conducted in this study, the cells were fixed after a 21-day period of induction of osteogenic differentiation using $4 \%$ paraformaldehyde for fifteen minutes. The cells were then stained with $1 \%$ ARS ( $\mathrm{pH} 4.2$, Sigma-Aldrich) for five minutes. Then, the staining solution was dissolved with a $10 \%$ cetylpyridinium chloride solution and quantified using an enzyme-labeled instrument (Thermo Fisher Scientific) at $562 \mathrm{~nm}$.

In regard to this study's oil red 0 staining process, after a 14-day period of induction of adipogenic differentiation, the cells were subjected to oil red O staining (Sigma). During the process, the cells were first fixed with $4 \%$ paraformaldehyde for thirty minutes, and then stained with an oil red 0 staining working solution (saturated oil red 0 and distilled water at a ratio of 3:2) for ten minutes. The lipid drops were shown as bright red under microscopy. Following the observational process, the staining solutions were dissolved with isopropanol and quantified using an enzyme-labeled instrument (Thermo Fisher Scientific) at $450 \mathrm{~nm}$.

\section{Quantifications of the global $\mathrm{m}^{6} \mathrm{~A}$ levels}

The global $\mathrm{m}^{6} \mathrm{~A}$ levels of the total RNA were measured using an EpiQuik $\mathrm{m}^{6} \mathrm{~A}$ RNA Methylation Quantification Kit (Colorimetric) (Epigentek) ${ }^{[22]}$, following the manufacturer's protocol. In this study, 200 ng of poly-A-purified RNA were used for each sample analysis process. The $\mathrm{m}^{6} \mathrm{~A}$ levels were quantified using a colorimetric method, and the absorbance of each well at a wavelength of $450 \mathrm{~nm}$ was measured. 
The calculations were completed in accordance with the standard curve reflected by the $\mathrm{m}^{6} \mathrm{~A}$ levels ${ }^{[23]}$. In addition, in order to ensure the accuracy of the results, each sample was run in triplicate.

\section{Analysis results of the RNA isolations and quantitative reverse transcription polymerase chain reactions (RT-qPCR)}

In the current study, the total RNAs were extracted from the HD-MSCs and AML-MSCs using a Trizol reagent (Invitrogen, Carlsbad, CA), during which the operational instructions were strictly followed. The purity levels and concentrations were measured using a NanoDrop 2000 spectrometer (Thermo Fisher Scientific, Waltham, MA). The analyses of the reverse transcription reactions were carried out using a cDNA synthesis kit (Roche, Mannheim, Germany). Furthermore, quantitative real-time PCR was performed according to the manufacturer's instructions, utilizing ABI 7500 Real-Time System (Life Technologies) and SYBR Green Fluorescence (Roche, Mannheim, Germany) instruments. The qPCR reaction components comprised $1 \mu \mathrm{l}$ of cDNA (with a 2.5 -fold dilution); $5 \mu$ of thunderbird SYBR Green qPCR mix; $0.3 \mu \mathrm{l}$ of PCR primers; and $3.4 \mu \mathrm{l}$ of RNase-free water. Next, the following protocol was undertaken: $95^{\circ} \mathrm{C}$ for 1 minute, and 35 cycles of $95^{\circ} \mathrm{C}$ for 15 seconds, $60^{\circ} \mathrm{C}$ for 30 seconds, and $72^{\circ} \mathrm{C}$ for 30 seconds, respectively. For the in-vitro experiments, the relative expressions were analyzed using a $2^{-\Delta \Delta \mathrm{Ct}}$ method. Also, in regard to the clinical data, the relative expressions were analyzed using a $2^{-\Delta C t}$ method and the GAPDH were used as references ${ }^{[24]}$. The primers used are listed in Table 1, and each sample was run in triplicate.

\section{Western Blotting Results}

The MSCs were collected to extract the proteins and the protein concentrations were determined using a BSA method. The denatured proteins were loaded and separated by $10 \%$ SDS-PAGE and transferred to polyvinylidene fluoride membranes. The membranes were blocked with $5 \%$ no-fat milk (Bio-rad) and then incubated with primary and secondary antibodies. The Western Blotting substrates were detected using an immuno-blotting process with Pierce ECL (Thermo Fisher Scientific). The antibodies used for the Western Blotting were as follows: METTL3 (86132, Cell Signaling Technology); AKT (12319, Cell Signaling Technology); p-AKT (13987, Cell Signaling Technology); and GAPDH (2118, Cell Signaling Technology), among which the GAPDH were used as references.

\section{Plasmid construction processes}

The wild-type METTL3-CDS were PCR-amplified from the HD-MSCs cDNA using the primers described in Table 1. The PCR products were purified and then cloned into modified pLJM1-EGFP (\#19319, Addgene) lentiviral vectors. The sequences of the shRNAs targeting human METTL3 were shRNA \#9 and shRNA $\# 12$, and the control scramble shRNA (Addgene \#1864) were purchased from Addgene ${ }^{[16]}$.

\section{Virus preparation and infection processes}


The recombinant lentivirus plasmids were extracted using an Endotoxin-free Plasmid Extraction Kit (QIAGEN) and co-transfected into 293 T cells with lentivirus packaging plasmids (psPAX2 and pMD2.G) for virus particles. Then, the virus particles were tested at 48 and 72 hours after transfection and concentrated with titer $\geq 1 \times 10^{8} \mathrm{TU} / \mathrm{ml}$. Subsequently, the virus was added to the MSCs and purinomycin for three days. Finally, the genomic DNA was extracted for verification purposes.

\section{RNA sequencing and data analyses of the MSCs}

RNA samples were extracted, and the concentrations were measured using NanoDrop 2000. Then, 1 to 2 ug of the total RNA from each sample were selected for the purpose of constructing an RNA sequencing library. The specific process was as follows: 1. Total RNA samples which were enriched in oligo dT (without rRNA) were selected; 2 . The samples were constructed using a KAPA Stranded RNA Sequencing Library Prep Kit (Illumina); 3 . During the library construction processes, double-strand cDNA was synthesized and combined with the subsequent high-fidelity PCR polymerase using a dUTP method, resulting in the RNA sequencing library having chain specificity; 4 . The constructed library was identified using an Alient 2100 Bioanalyzer and quantified by qPCR; 5 . The mixed sample libraries were sequenced using Illumina X-ten/NovaSeq.

The image processing and base recognition were carried out in this study by adopting the software of Solexa Pipeline Version 1.8. In addition, FastQ software was utilized to evaluate the sequencing quality of the reads (using Cutsapt to remove $3^{\prime}$ and 5 ' splices). Then, $\mathrm{R}$ software Balltown ${ }^{[25,26]}$ was used to calculate the FPKM (fragments per kilobase of gene/transcription model per million mapped fragments) at the transcriptional level, as well as calculate and screen the differentially expressed genes. The threshold was set to a 1.5 -fold change, $p$-value $\leq 0.05$, and mean value of the FPKM in the group $\geq 0.5$, in order to screen the differentially expressed genes. Finally, the results of the GO (gene ontology) functional significance enrichment analysis processes, as well as the results of the KEGG (Kyoto Encyclopedia of genes and genomes) pathway significance enrichment analysis processes, were further analyzed in this study.

\section{Microarray hybridization and data analysis results}

The immuno-precipitated RNA samples from the HD-MSCs and AML-MSCs were labeled with Cy5 fluorescent dye using a Super RNA Labeling Kit (Arraystar), and then purified using an RNeasy Mini Kit. The Cy 5 labeled cRNAs were fragmented and hybridized to human mRNA and IncRNA $\mathrm{m}^{6} \mathrm{~A}$ epitranscriptomic microarray ( $8 \times 60 \mathrm{~K}$, Arraystar), which contained 44,122 mRNAs and 12,496 IncRNAs degenerate probes. The hybridized arrays were scanned using an Agilent Scanner G2505C [27].

This study utilized Agilent feature extraction software (Version 11.0.1.1), and each spot on a microarray was evaluated and flagged as present, absent, or marginal, based on its uniformity above the background and saturation levels. The raw intensities of the immuno-precipitated RNAs were normalized with an average of log2-scaled spike-in RNA intensities, and the fold changes and $P$ values were calculated for each transcript between the HD-MSCs and the AML-MSCs. A cutoff of 5 -fold $(P<0.05)$ was used to 
identify the differentially $\mathrm{m}^{6} \mathrm{~A}$ methylated RNAs. At that point in the experiment, both $\mathrm{GO}$ analysis and KEGG Pathway analysis were performed for the differentially $\mathrm{m}^{6} \mathrm{~A}$ methylated $\mathrm{mRNA}$ transcripts using the DAVID (Database for Annotation, Visualization, and Integrated Discovery) bioinformatics database ${ }^{[28]}$.

\section{SRAMP database}

SRAMP (which is based on RNA adenosine methylation site predictions) is a sequence-based $\mathrm{m}^{6} \mathrm{~A}$ modification site predictor ${ }^{[29]}$. It has been found that SRAMP can be a useful tool for predicting $\mathrm{m}^{6} \mathrm{~A}$ modification sites on RNA sequences of interest. It was required to input the full-length RNA sequence of the AKT into the prediction website in order to predict the $\mathrm{m}^{6} \mathrm{~A}$ modification site on the sequence according to the specific $\mathrm{m}^{6} \mathrm{~A}$ modification sequence.

\section{Statistical analysis results}

In this study, the experiments were repeated three times for all of the examined specimens. Statistical analysis was performed using GraphPad Prism8.0 software, and the obtained statistical differences between the groups were determined using a Student's $t$ test. In the current study, P-values $<0.05$ were considered to be statistically significant.

\section{Results}

\section{Significant increases in the adipogenic differentiations of the AML-MSCs}

The detections of cell morphology, phenotype, and multidirectional differentiation potential of the MSCs indicated that there were no differences in the HD-MSCs and the AML-MSCs (Supplementary Fig. 1A to 1D). During the cell culturing process, there were also no significant differences observed in proliferation between the HD-MSCs and AML-MSCs (Supplementary Fig. 1E). The adipogenic differentiations of the HD-MSCs/AML-MSCs were induced, and the adipocyte was detected using an oil red 0 staining process, as shown in Fig. 1A. Then, after the isopropanol was dissolved, the OD450 was determined (Fig. 1B). The results indicated that the adipogenic differentiation ability of the AML-MSCs was stronger than that of the HD-MSCs, and the survival timeframes of the AML patients with high adipogenic differentiations would be significantly shorter than those of patients with low adipogenic differentiations, as illustrated in Fig. 1C.

\section{AML-MSCs inhibit the chemo-resistance of AML cells more significantly than HD-MSCs following the induction of adipogenic differentiations}

In the current study, the adipogenic differentiations of HD-MSCs and AML-MSCs were induced for fourteen days, and then co-cultured with AML cells in order to test the chemo-resistance of the AML cells. The results showed that the chemo-resistance of the AML-MSCs to AML cells (including HL-60, U937 and THP-1) were significantly higher than that of the HD-MSCs, as detailed in Fig. 2. Therefore, it was indicated that they may play important roles in affecting the chemo-resistance of AML. Subsequently, it 
was particularly important in this study to identify the molecular mechanism affecting the adipogenic differentiations.

\section{Global $\mathrm{m}^{6} \mathrm{~A}$ levels and expressions of METTL3 decreased in the AML-MSCs}

It has been determined that RNA epigenetic modifications play key roles in the differentiations of stem cells. The results of previous experimental studies have shown that $\mathrm{m}^{6} \mathrm{~A}$ methylase METTL3 played a key role in the processes of adipogenic differentiation of bone MSCs in pigs ${ }^{[30]}$ and mouse ${ }^{[31]}$. Therefore, confirming that $\mathrm{m}^{6} \mathrm{~A}$ modifications play important roles in the differentiations of human bone marrow MSCs was a priority in the present study. An EpiQuik m6 $\mathrm{A}$ RNA methylation quantitative detection kit (Epigentek) was adopted to detect the global $\mathrm{m}^{6} \mathrm{~A}$ levels. The results showed that when compared with the HD-MSCs, the $\mathrm{m}^{6} \mathrm{~A}$ levels of total RNA in the AML-MSCs were decreased (Fig. $3 \mathrm{~A}$ ). Meanwhile, the results of the RT-qPCR (Fig. 3B) and Western Blot (Fig. 3C) showed that the expressions of METTL3 were significantly decreased in the AML-MSCs. The next step was to determine if the increased adipogenic differentiation abilities of the AML-MSCs were related to the expressions of METTL3. In order to verify the effects of the METTL3 on the adipogenic differentiations of the MSCs, this study induced the adipogenic differentiations of the MSCs by overexpression and knockdown of the METTL3. Subsequently, the changes in the adipogenic differentiation abilities of the MSCs were successfully detected. The results indicated that following the infection of the AML-MSCs with METTL3 overexpression lentivirus, the RTqPCR (Fig. 4A) and Western Blot (Fig. 4B) results showed significant overexpressions. In addition, the results revealed that the METTL3 overexpressions could significantly inhibit the adipogenic differentiations of the AML-MSCs (Fig. 4C). In contrast, two independent shRNAs targeting METTL3 were used to knockdown the METTL3 in the HD-MSCs, and the RT-qPCR (Fig. 4D) and Western Blot (Fig. 4E) results had verified the significant knockdown. The adipogenic differentiations of the HD-MSCs were found to be significantly enhanced (Fig.4F). Therefore, it was confirmed that the METTL3 was a key factor in the regulation of the adipogenic differentiations of the MSCs. However, the question still remained as to how the $\mathrm{m} 6 \mathrm{~A}$ regulates the expressions of target genes and affects the adipogenic differentiations of the MSCs. Therefore, the mRNA expressions and differential m6A expressions of the HD-MSCs and AML-MSCs were detected in this study in order to further explore the intrinsic mechanism of adipogenic differentiations of the MSCs.

\section{High expressions of AKT in the AML-MSCs and their association with the adipogenic differentiations}

In order to access the differentially expressed genes between the HD-MSCs and AML-MSCs, this study sequenced the MSCs RNA of three HD and four untreated samples of newly diagnosed AML. In this study, all of the subjects were ranged between 30 and 50 years of age, and the samples were sequenced from culture to the third generation. The results of the principle component analysis (PCA) showed distinct clustering in the individual samples of HD-MSCs and AML-MSCs (Fig. 5A), indicating the rigor and reproducibility of the samples. The results of the sequence analysis showed that 1,069 genes, including 242 up-regulated genes and 828 down-regulated genes, were significantly different between the two groups (|fold changel $\geq 1.0$; P-value < 0.05), and were displayed in the form of a volcanic map (Fig. 5B). 
Then, based on the differentially expressed genes, gene enrichment analysis was carried out. The results of the GO analysis showed that, when compared with the HD-MSCs, the AML-MSCs were significantly upregulated in terms of certain biological processes, including the regulation of signaling receptor activities; blood vessel morphogenesis; extracellular matrix organization; extracellular structure organization; and so on (Fig. 5C), which indicated that the MSCs may change the microenvironmental conditions through signal exchanges between the extracellular matrix components and the extracellular substances. This study's KEGG analysis results indicated that the PI3K/AKT signal pathways were significantly upregulated in the AML-MSCs, and may potentially be involved in the processes of enhancing the adipogenic differentiation abilities of the AML-MSCs (Fig. 5D). In addition, RT-qPCR and Western Blot were used to detect the expression levels of the AKT mRNA and proteins in the examined clinical samples. The results showed that the AKT activities and expression levels in the AML-MSCs were significantly up-regulated (Figs. 5E and 5F). It has been noted that the AKT inhibitor MK-2206 2HCL is a highly selective AKT1/2/3 inhibitor, which has the ability to inhibit AKT activities in AML-MSCs and induce the adipogenic differentiations of the MSCs. The results obtained in this study indicated that the adipogenic differentiations of the MSCs with MK-2206 2HCL were significantly inhibited, as illustrated in Fig. 5G.

\section{Significantly reduced $\mathrm{m}^{6} \mathrm{~A}$ levels of AKT mRNA in the AML-MSCs}

This study analyzed the methylation RNAs of $\mathrm{m}^{6} \mathrm{~A}$ in the HD-MSCs and AML-MSCs, and HD-MSCs served as the control. The methylation RNAs were profiled using the microarray with probes for 44,122 mRNAs and 12,496 IncRNAs. The results of microarray profiling showed that 127 mRNAs were lowly methylated between the HD-MSCs and AML-MSCs (Fig. 6A). In addition, this study's KEGG (Fig. 6B) and GSEA (Fig. $6 C$ ) analysis results showed that, when compared with the HD-MSCs, the $\mathrm{m}^{6} \mathrm{~A}$ levels of the PI3K/AKT signaling pathways in the AML-MSCs were significantly reduced. This study also used the online prediction to mammal $\mathrm{m}^{6} \mathrm{~A}$ modification site website SRAMP to predict the $\mathrm{m}^{6} \mathrm{~A}$ modification sites of the AKT. The predicted results showed that for a total forecast of $27 \mathrm{~m}^{6} \mathrm{~A}$ modification sites (Fig. 6D), eight were very high confidence $\mathrm{m}^{6} \mathrm{~A}$ sites; seven were high confidence $\mathrm{m}^{6} \mathrm{~A}$ sites; four were moderate confidence $\mathrm{m}^{6} \mathrm{~A}$ sites; and three were low confidence $\mathrm{m}^{6} \mathrm{~A}$ sites, as detailed in Table 2. Further experiments will be carried out on the aforementioned sites.

\section{Discussion}

It is known that $\mathrm{m}^{6} \mathrm{~A}$ methylation modifications play a key role in regulating the pluripotency, proliferation, metastasis, and immunity of tumor stem cells. METTL3, which is a type of $\mathrm{m}^{6} \mathrm{~A}$ methylase, has been observed to have two-way effects in many types of cancers. METTL3 is highly expressed in AML $[16,32$, ${ }^{33]}$, liver cancer ${ }^{[22]}$, glioblastoma ${ }^{[34]}$, and other tumors, and can promote the occurrence and development of tumors. In contrast, it has been reported that METTL3 can also be used as a tumor suppressor to inhibit the growth and invasion of ovarian cancer ${ }^{[35]}$ and prostate cancer ${ }^{[36]}$. However, there have been few studies conducted regarding MSC. However, high expressions have been found to 
inhibit the adipogenic differentiations of MSC in healthy pigs and mice. At the present time, the effects on human MSCs have not yet been reported.

AML bone marrow microenvironments are continuously remodeled to ensure AML cells survive and escape the negative effects of chemotherapy. The adipocytes in bone marrow are mainly differentiated from MSCs, and the adipogenic differentiations of MSCs in AML bone marrow are enhanced, which provides strong support for the killing of AML cells through chemotherapy. However, the intrinsic molecular mechanism of enhanced adipogenic differentiations of AML-MSCs remains elusive. The modifications of $\mathrm{m}^{6} \mathrm{~A}$ have been found to be the most abundant RNA modifications on eukaryotic mRNA and are important factors affecting stem cell differentiation. At the present time, the role of $m R N A-m^{6} A$ in tumor genesis and development has been seldom investigated from the perspective of tumor microenvironmental MSCs. The preliminary results of this study demonstrated that, when compared with the HD-MSCs, the AML-MSCs exhibited decreased expressions of METTL3. Moreover, the expressions of METTL3 were determined to be significantly related to the adipogenic differentiations of the MSCs. The results of the RNA sequencing and $\mathrm{m}^{6} \mathrm{~A}$ microarray demonstrated that the activities of the $\mathrm{m}^{6} \mathrm{~A}$ levels in the PI3K/AKT signaling pathways were reduced. The $\mathrm{m}^{6} \mathrm{~A}$ of PI3K/AKT signaling pathways were observed to be significantly up-regulated, which may have promoted the adipogenic differentiations of the MSCs. However, the precise molecular mechanisms will require further exploration. The results of previous related studies have shown that decreases in METTL3 regulated AKT activities promoted the proliferation and tumorigenicity of endometrial cancer, and m6A methylation had been confirmed to be a regulator of the AKT pathways ${ }^{[37]}$. However, there have not yet been reports made available regarding the regulations of PI3K/AKT signaling pathways by METTL3 expressions in the adipogenic differentiations of MSCs.

The results of the differentially expressed genes in this study's RNA sequencing showed that the lower expressions of the AML-MSCs mRNA gene numbers were more than the high expressions. In addition, the results of the $\mathrm{m}^{6} \mathrm{~A}$ microarray showed that the low m6A modified mRNA gene numbers were greater than that of the high m6A modified genes in the AML-MSCs. Therefore, based on the fact that the $\mathrm{m}^{6} \mathrm{~A}$ functions mainly depend on the "readers", this study speculated that IGF2BP proteins ${ }^{[38]}$ may play important roles in subsequent functions. Also, according to the KEGG analysis of the RNA sequencing and the m6A microarray, it was determined that only the PI3K/AKT signaling pathways were characterized by low levels of $\mathrm{m}^{6} \mathrm{~A}$, while the transcription levels were significantly increased. Therefore, the PI3K/AKT signaling pathways modified by m6A during the process of adipogenic differentiation may function by interacting with YTHDF2 ${ }^{[39]}$, which will be further verified in the following experiments. At the present time, there are many m6A modification site prediction websites, such as MeT-BD [40] and WISTLE ${ }^{[41]}$, which are based on SRAMP, and are not widely applied as SRAMP. The SRAMP prediction site predicted different confidence sites based on the characteristic motif sequence of the $\mathrm{m}^{6} \mathrm{~A}$. Of course, whether or not the predicted sites are accurate still requires verification by experimental data. 


\section{Conclusions}

In summary, our research demonstrated that METTL3 is highly related to the adipogenic differentiations of bone marrow MSCs. It was observed that decreased expressions of METTL3 in the AML-MSCs could significantly reduce the level of $\mathrm{m}^{6} \mathrm{~A}$ modification of the PI3K/AKT signaling pathways. Furthermore, consequent activations of the PI3K/AKT signaling pathways could promote the adipogenic differentiations of MSCs, which could potentially mediate the chemo-resistance of AML (Fig. 7). The results obtained in this research will provide a theoretical basis for determining new targets of AML treatment from the perspective of bone marrow microenvironments, which will potentially have important clinical application value in the future.

\section{Abbreviations}

AML: Acute Myeloid Leukemia; MSCs: Mesenchymal Stem Cells; HD-MSCs \Mesenchymal Stem Cells from Healthy Donors; AML-MSCs: Mesenchymal Stem Cells from Acute Myeloid Leukemia; METTL3: Methyltransferase Like 3; METTL14: Methyltransferase Like 14; WTAP: WT1 Associated Protein; FTO: Fat mass and obesity associated protein; ALKBH5: alkB homolog 5; $\mathrm{m}^{6} \mathrm{~A} \llbracket \mathrm{N}^{6}$-methyladenine; BMM: Bone Marrow Microenvironments; FBS: Fetal Bovine Serum; mRNA: messenger RNA; PI3K: Phosphatidylinositol 3-kinase; AKT: Protein kinase B; GAPDH: Glyceraldehyde3-phosphate dehydrogenase; CCK-8: Cell Counting Kit-8; MNC: Mononuclear Cells; ARS: Alizarin Red Staining; RT-qPCR: Reverse Transcription Polymerase Chain Reactions; CDS: Coding Sequence; GO: Gene Ontology; KEGG :Kyoto Encyclopedia of Genes and Genomes; IncRNA囚Long non-coding RNA; DAVID: Database for Annotation, Visualization, and Integrated Discovery; PCA: Principle Component Analysis; GSEA: Gene Set Enrichment Analysis; IGF2BP: Insulin like growth factor 2 mRNA Binding Protein; YTHDF2: YTH N6-methyladenosine RNA Binding Protein 2; SDS-PAGE: Sodium Dodecyl Sulfate Polyacrylamide Gel Electrophoresis; shRNA: short hairpin RNA

\section{Declarations}

\section{Acknowledgements}

Not applicable

\section{Authors' contributions}

Zhipeng Pan, Bin Wang, Diyu Hou, Ruolan You, and Xiaoting Wang performed the experiments. Wenhui Xie analyzed data and Zhipeng Pan wrote the manuscript. Huifang Huang designed the experiments. The authors read and approved the final manuscript.

\section{Funding}


This research was supported by the National Natural Science Foundation of China (81870108); Fujian Medicine Innovation Program (2018-CX-18); Joint Funds for the innovation of Science and Technology, Fujian Province (2017Y9053); Government-funded projects for the construction of high-level laboratories and the construction project of the Fujian Medical Center of Hematology (Min201704); and the National and Fujian Provincial Key Clinical Specialty Discipline Construction Program of the PRC.

\section{Availability of data and materials}

The datasets used and/or analyzed during the current study are available from the corresponding author on reasonable request.

\section{Ethics approval and consent to participate}

All clinical specimens were conducted in accordance with standard operating procedures under the consent and guidance of the Ethics Committee of the Fujian Medical University Union Hospital. Consent to participate is not applicable.

\section{Consent for publication}

Not applicable

\section{Competing interests}

The authors declare that they have no competing interests.

\section{References}

1. F Ferrara, Lancet Schiffer. Acute myeloid leukaemia in adults. Lancet (London, England), 2013, 381(9865):484-95.

2. Khwaja A., Bjorkholm M., Gale R. E., et al. Acute myeloid leukaemia. Nat Rev Dis Primers, 2016, 2:16010.

3. Korn C., Mendez-Ferrer S. Myeloid malignancies and the microenvironment. Blood, 2017, 129(7):81122.

4. Witkowski M. T., Kousteni S., Aifantis I. Mapping and targeting of the leukemic microenvironment. J Exp Med, 2020, 217(2).

5. Medyouf $\mathrm{H}$. The microenvironment in human myeloid malignancies: emerging concepts and therapeutic implications. Blood, 2017, 129(12):1617-26.

6. Rauch A., Haakonsson A. K., Madsen J. G. S., et al. Osteogenesis depends on commissioning of a network of stem cell transcription factors that act as repressors of adipogenesis. Nat Genet, 2019, 51(4):716-27.

7. Azadniv M., Myers J. R., McMurray H. R., et al. Bone marrow mesenchymal stromal cells from acute myelogenous leukemia patients demonstrate adipogenic differentiation propensity with implications 
for leukemia cell support. Leukemia, 2020, 34(2):391-403.

8. Geyh S., Rodriguez-Paredes M., Jager P., et al. Functional inhibition of mesenchymal stromal cells in acute myeloid leukemia. Leukemia, 2016, 30(3):683-91.

9. MR Reagan, Rheumatology Rosen CJ \%J Nature reviews. Navigating the bone marrow niche: translational insights and cancer-driven dysfunction. 2016, 12(3):154-68.

10. Wu Y., Zhou C., Yuan Q. Role of DNA and RNA N6-Adenine Methylation in Regulating Stem Cell Fate. Curr Stem Cell Res Ther, 2018, 13(1):31-38.

11. Wu J., Frazier K., Zhang J., et al. Emerging role of m(6) A RNA methylation in nutritional physiology and metabolism. Obes Rev, 2020, 21(1):e12942.

12. Roundtree I. A., Evans M. E., Pan T., et al. Dynamic RNA Modifications in Gene Expression Regulation. Cell, 2017, 169(7):1187-200.

13. Y Yang, PJ Hsu, YS Chen, et al. Dynamic transcriptomic mA decoration: writers, erasers, readers and functions in RNA metabolism. Cell Res, 2018, 28(6):616-24.

14. Wang H., Hu X., Huang M., et al. Mett|3-mediated mRNA m(6)A methylation promotes dendritic cell activation. Nat Commun, 2019, 10(1):1898.

15. Vu L. P., Cheng Y., Kharas M. G. The Biology of m(6)A RNA Methylation in Normal and Malignant Hematopoiesis. Cancer Discov, 2019, 9(1):25-33.

16. Vu L. P., Pickering B. F., Cheng Y., et al. The N(6)-methyladenosine (m(6)A)-forming enzyme METTL3 controls myeloid differentiation of normal hematopoietic and leukemia cells. Nat Med, 2017, 23(11):1369-76.

17. Li Z., Weng H., Su R., et al. FTO Plays an Oncogenic Role in Acute Myeloid Leukemia as a N(6)Methyladenosine RNA Demethylase. Cancer Cell, 2017, 31(1):127-41.

18. Su R., Dong L., Li C., et al. R-2HG Exhibits Anti-tumor Activity by Targeting FTO/m(6)A/MYC/CEBPA Signaling. Cell, 2018, 172(1-2):90-105 e23.

19. Zhang L., Lei Q., Wang H., et al. Tumor-derived extracellular vesicles inhibit osteogenesis and exacerbate myeloma bone disease. Theranostics, 2019, 9(1):196-209.

20. Huang R. L., Sun Y., Ho C. K., et al. IL-6 potentiates BMP-2-induced osteogenesis and adipogenesis via two different BMPR1A-mediated pathways. Cell Death Dis, 2018, 9(2):144.

21. Li Y., Liu Z., Tang Y., et al. Schnurri-3 regulates BMP9-induced osteogenic differentiation and angiogenesis of human amniotic mesenchymal stem cells through Runx2 and VEGF. Cell Death Dis, 2020, 11(1):72.

22. Chen M., Wei L., Law C. T., et al. RNA N6-methyladenosine methyltransferase-like 3 promotes liver cancer progression through YTHDF2-dependent posttranscriptional silencing of SOCS2. Hepatology, 2018, 67(6):2254-70.

23. Ma J. Z., Yang F., Zhou C. C., et al. METTL14 suppresses the metastatic potential of hepatocellular carcinoma by modulating N(6) -methyladenosine-dependent primary MicroRNA processing. Hepatology, 2017, 65(2):529-43. 
24. Li X. C., Jin F., Wang B. Y., et al. The m6A demethylase ALKBH5 controls trophoblast invasion at the maternal-fetal interface by regulating the stability of CYR61 mRNA. Theranostics, 2019, 9(13):385365.

25. Frazee A. C., Pertea G., Jaffe A. E., et al. Ballgown bridges the gap between transcriptome assembly and expression analysis. Nat Biotechnol, 2015, 33(3):243-6.

26. Pertea M., Kim D., Pertea G. M., et al. Transcript-level expression analysis of RNA-seq experiments with HISAT, StringTie and Ballgown. Nat Protoc, 2016, 11(9):1650-67.

27. Chokkalla A. K., Mehta S. L., Kim T., et al. Transient Focal Ischemia Significantly Alters the m(6)A Epitranscriptomic Tagging of RNAs in the Brain. Stroke, 2019, 50(10):2912-21.

28. Dennis G., Jr., Sherman B. T., Hosack D. A., et al. DAVID: Database for Annotation, Visualization, and Integrated Discovery. Genome Biol, 2003, 4(5):P3.

29. Zhou Y., Zeng P., Li Y. H., et al. SRAMP: prediction of mammalian N6-methyladenosine (m6A) sites based on sequence-derived features. Nucleic Acids Res, 2016, 44(10):e91.

30. Yao Y., Bi Z., Wu R., et al. METTL3 inhibits BMSC adipogenic differentiation by targeting the JAK1/STAT5/C/EBPbeta pathway via an m(6)A-YTHDF2-dependent manner. FASEB J, 2019, 33(6):7529-44.

31. Wu Y., Xie L., Wang M., et al. Mett|3-mediated m(6)A RNA methylation regulates the fate of bone marrow mesenchymal stem cells and osteoporosis. Nat Commun, 2018, 9(1):4772.

32. Barbieri I., Tzelepis K., Pandolfini L., et al. Promoter-bound METTL3 maintains myeloid leukaemia by m(6)A-dependent translation control. Nature, 2017, 552(7683):126-31.

33. Wang X. S., He J. R., Yu S., et al. [Methyltransferase-like 3 Promotes the Proliferation of Acute Myeloid Leukemia Cells by Regulating N6-methyladenosine Levels of MYC]. Zhongguo Yi Xue Ke Xue Yuan Xue Bao, 2018, 40(3):308-14.

34. Cui Q., Shi H., Ye P., et al. m(6)A RNA Methylation Regulates the Self-Renewal and Tumorigenesis of Glioblastoma Stem Cells. Cell Rep, 2017, 18(11):2622-34.

35. Li X., Tang J., Huang W., et al. The M6A methyltransferase METTL3: acting as a tumor suppressor in renal cell carcinoma. Oncotarget, 2017, 8(56):96103-16.

36. Taketo K., Konno M., Asai A., et al. The epitranscriptome m6A writer METTL3 promotes chemo- and radioresistance in pancreatic cancer cells. Int J Oncol, 2018, 52(2):621-29.

37. Liu J., Eckert M. A., Harada B. T., et al. m(6)A mRNA methylation regulates AKT activity to promote the proliferation and tumorigenicity of endometrial cancer. Nat Cell Biol, 2018, 20(9):1074-83.

38. Huang $\mathrm{H}$., Weng H., Sun W., et al. Recognition of RNA N(6)-methyladenosine by IGF2BP proteins enhances mRNA stability and translation. Nat Cell Biol, 2018, 20(3):285-95.

39. Zaccara S., Ries R. J., Jaffrey S. R. Reading, writing and erasing mRNA methylation. Nat Rev Mol Cell Biol, 2019, 20(10):608-24.

40. Liu H., Wang H., Wei Z., et al. MeT-DB V2.0: elucidating context-specific functions of N6-methyladenosine methyltranscriptome. Nucleic Acids Res, 2018, 46(D1):D281-D87. 
41. Chen K., Wei Z., Zhang Q., et al. WHISTLE: a high-accuracy map of the human N6-methyladenosine (m6A) epitranscriptome predicted using a machine learning approach. Nucleic Acids Res, 2019, 47(7):e41.

\section{Tables}

Table 1 Primer sequences of the genes

\begin{tabular}{|c|c|}
\hline Primers name & Primer sequence $\left(5^{\prime} \rightarrow 3^{\prime}\right)$ \\
\hline METTL3-PCR-F* & 5'- AAATCTAGAATGTCGGACACGTGGAGCTC-3' \\
\hline METTL3-PCR-R & 5'- TTTGCGATCGCCTATAAATTCTTAGGTTTAGAG -3' \\
\hline METTL3-qPCR-F & 5'- CAGGGCTGGGAGACTAGGAT-3' \\
\hline METTL3-qPCR-R & 5'- CTGGGCTGTCACTACGGAAG -3' \\
\hline AKT-qPCR-F & 5'-TGAGGAGCCCTGGTCTAATGAT-3' \\
\hline AKT-qPCR-R & 5'-AAGACCCATTCAGAAGAGTTAT-3' \\
\hline GAPDH-qPCR-F & 5'- GGAGCGAGATCCCTCCAAAAT-3' \\
\hline GAPDH-qPCR-R & 5'- GGCTGTTGTCATACTTCTCATGG-3' \\
\hline
\end{tabular}

*F: Forward primer

\#R: Reverse primer

Table 2 Predictions of the AKT m6A sites 


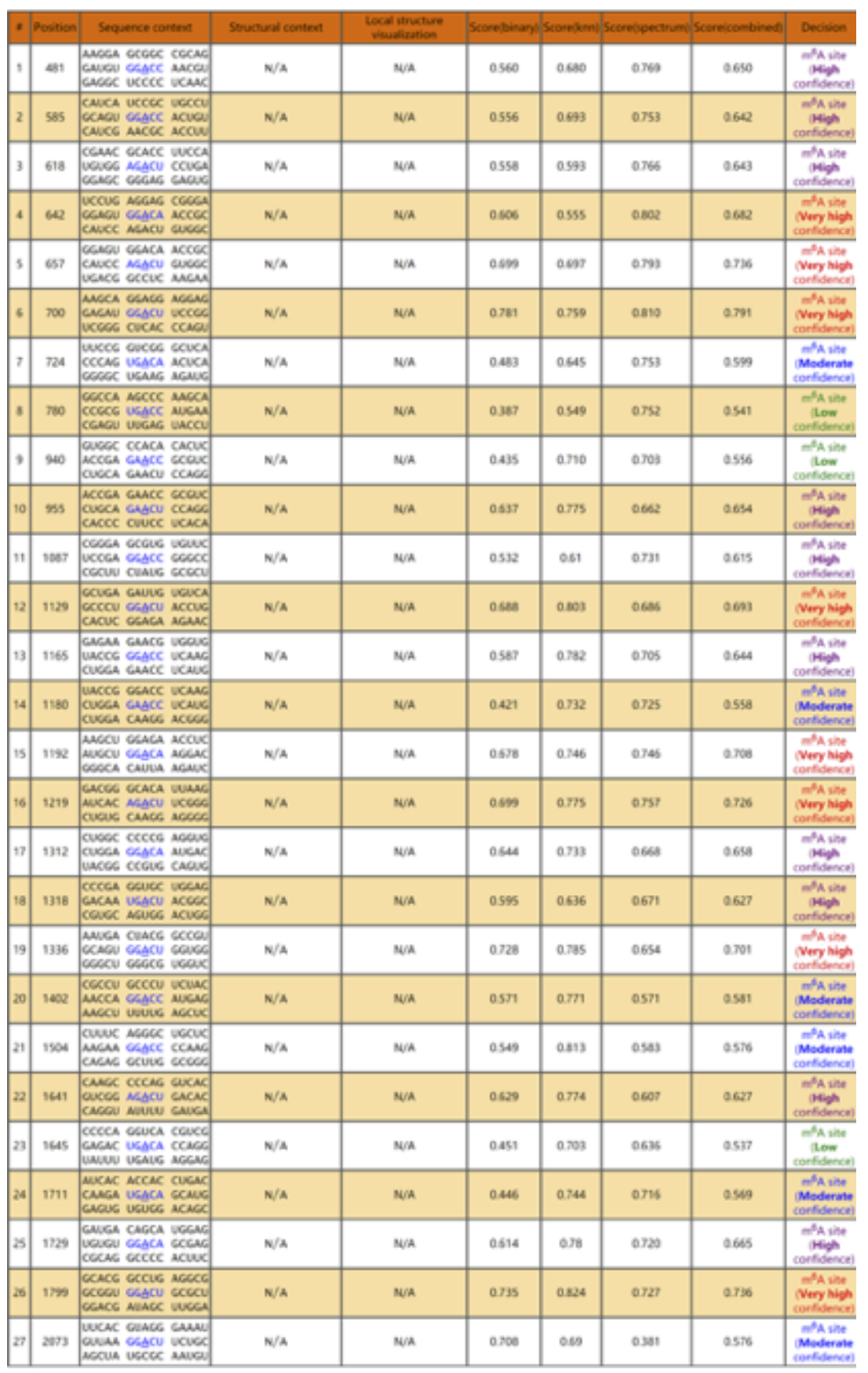

\section{Figures}
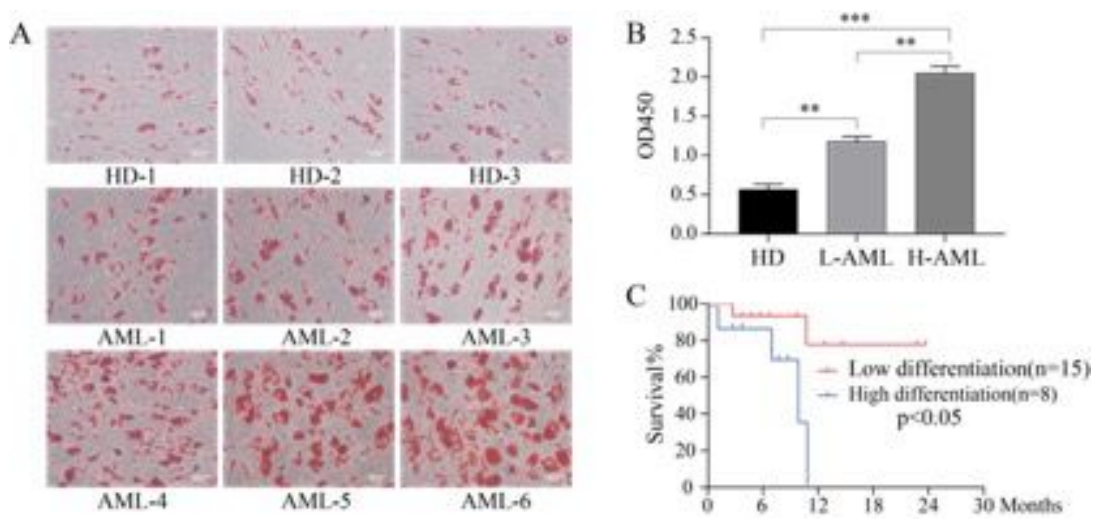


\section{Figure 1}

Adipogenic differentiation ability of bone marrow MSCs A. AML-MSCs/HD-MSCs after adipogenic differentiation were observed and photographed with oil red $\mathrm{O}$ staining; $\mathrm{B}$. Following the oil red $\mathrm{O}$ staining, the isopropanol dissolved lipid dropped at room temperature, and enzyme scale was used to detect the absorbance value at $450 \mathrm{~nm} ; \mathrm{C}$. Analysis of the survival times of AML patients: HD: healthy donor; OD 450: after MSCs are induced by adipogenic differentiation, it was treated with isopropanol to dissolve fat, and the absorbance value of $450 \mathrm{~nm}$ measured by an enzyme scale instrument indicated the degree of adipogenic differentiations (same as below); L-AML/Low differentiation AML: AML patients with low adipogenic differentiation of MSCs; H-AML/High differentiation AML: AML patients with high adipogenic differentiation of MSCs; Low differentiation: Low adipogenic differentiation; High differentiation: High adipogenic differentiation; Note: In the figure, ${ }^{*} \mathrm{P}<0.05$; ${ }^{\star \star} \mathrm{P}<0.01 ; \star \star \star \mathrm{P}<0.001$ (same as below)

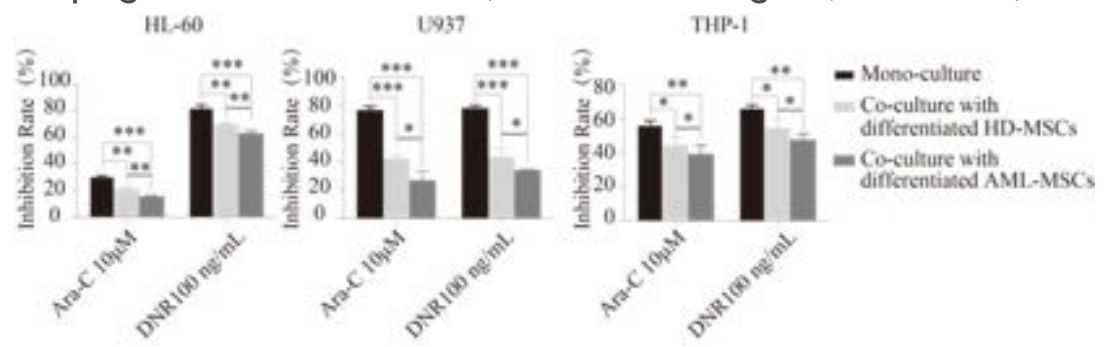

\section{Figure 2}

Effects of adipogenic differentiation of the MSCs on the chemo-resistance of the AML cells: After inducing adipogenic differentiation of HD-MSCs/AML-MSCs for 14 days, they were co-cultured with AML cells, and then detections of the chemo-resistance levels of the AML cells were completed in every group

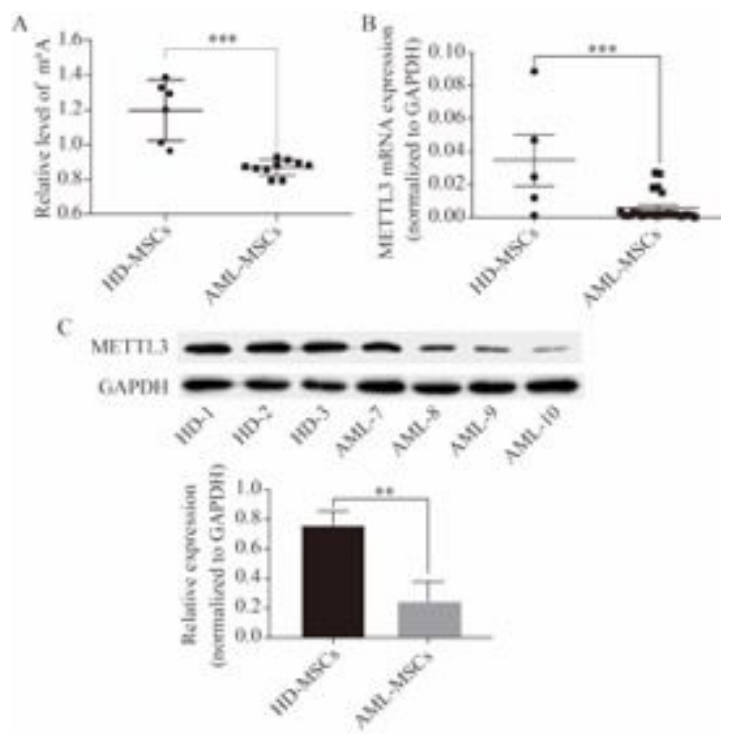

\section{Figure 3}

Potential for METTL3 inhibiting the adipogenic differentiations of MSCs. A. RT-qPCR to verify the mRNA levels of the METTL3 in AML-MSCs infected with METTL3 overexpression lentivirus; B. Western Blot to verify the METTL3 protein levels in the AML-MSCs infected with METTL3 overexpression lentivirus; C. 
After the induction of adipogenic differentiation, oil red $\mathrm{O}$ staining was observed by microscopy and the OD450 values were observed with an isopropanol room temperature solution; D. RT-qPCR to verify the mRNA level of METTL3 in the HD-MSCs after METTL3 knockdown; E. Western Blot to verify the protein levels of the METTL3 in the HD-MSCs after METTL3 knockdown; F. HD-MSCs to knock down METTL3 after adipogenic differentiation induction, oil red $\mathrm{O}$ staining under microscopy, and isopropanol room temperature lipolysis to detect OD450 (blank, wild strain); Control, empty body control; OE, overexpression; shRNA \#9 and shRNA \#12, two independent shRNAs targeting human METTL3.
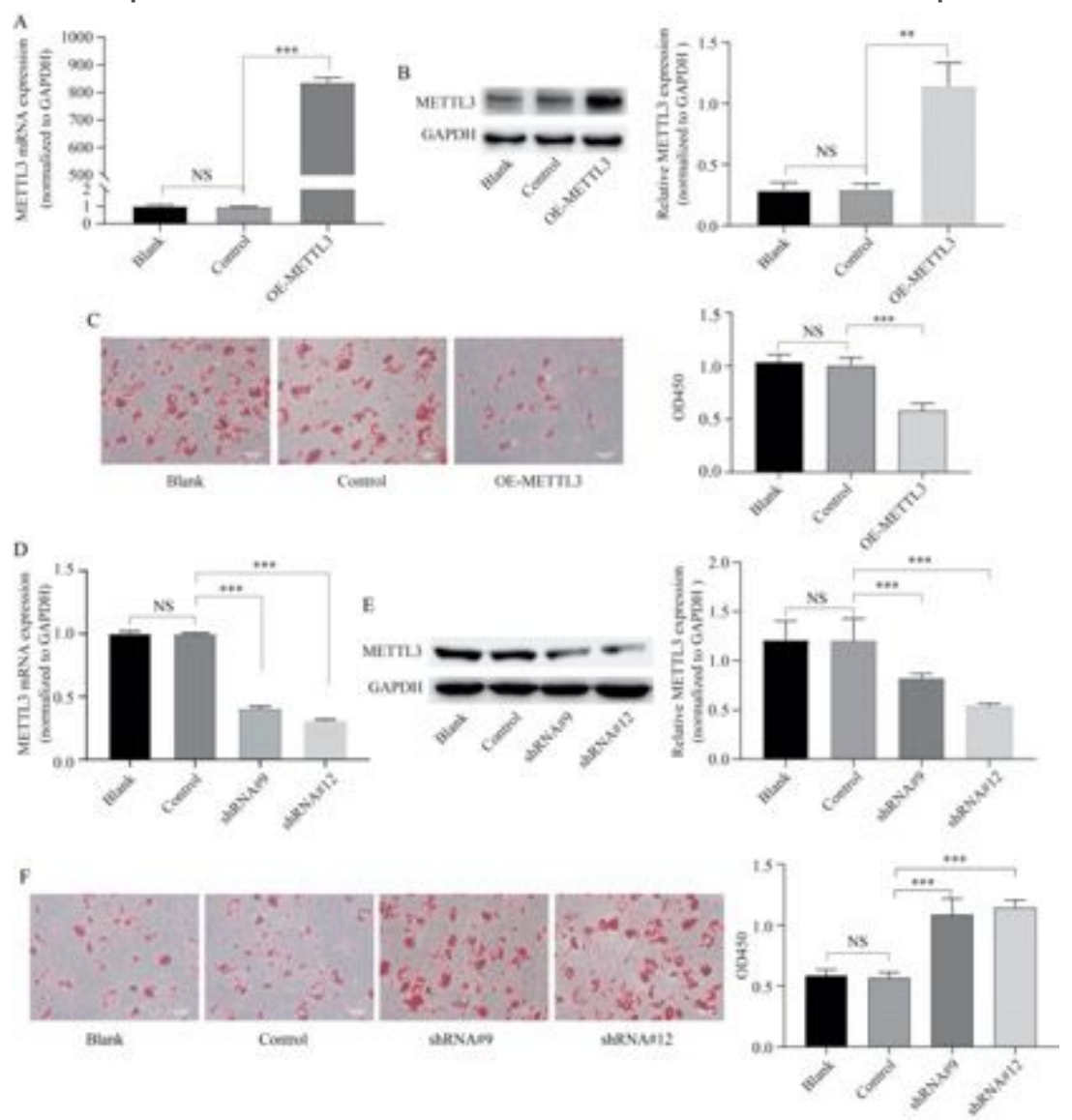

\section{Figure 4}

AKT highly expressed in AML-MSCs and associated with adipogenic differentiations. A. Principal component analysis of the transcription differences indicated that the AML-MSCs were significantly different from the HD-MSCs, and that the correlation variance percentages of the PC1 and PC2 were 58.5 and 10.8, respectively; B. Differential expression gene volcanic map; C. Biological process (BP) results in the GO gene enrichment analysis; D. KEGG analysis of the AML-MSCs and HD-MSCs at the level of transcription indicating significantly different pathways; E. RT-qPCR to verify the differences in the AKT mRNA levels between the AML-MSCs and HD-MSCs; F. Western Blot to detect the differences in the AKT total protein levels and activation levels of the p-AKT (ser473); G. AKT inhibitors to inhibit AKT activities in order to induce adipogenic differentiations and the results of isopropanol lipolysis 

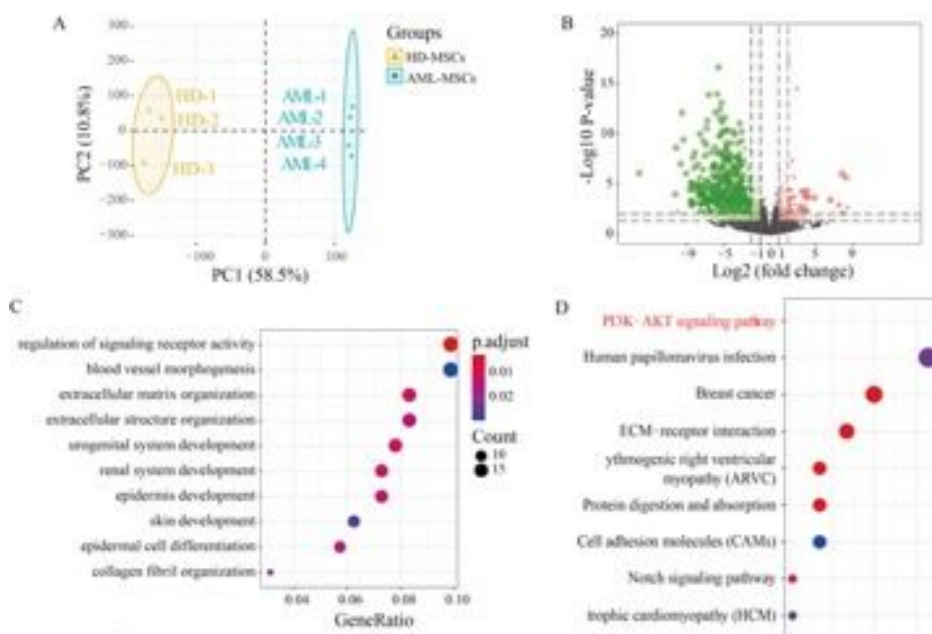

D
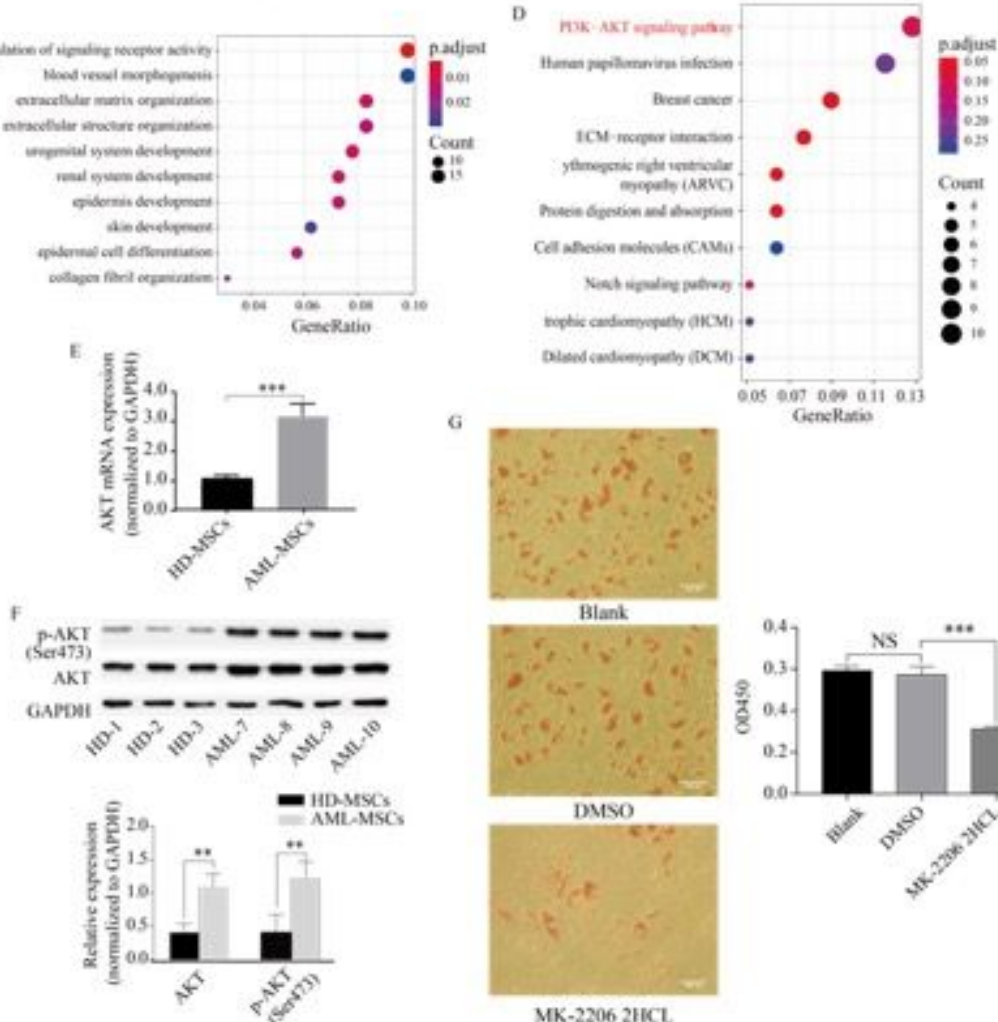

Geneliatio

MK-2206 2HICL

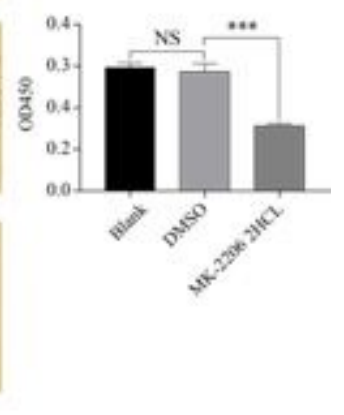

Figure 5

Global m6A levels and the decreased expressions of METTL3 in the AML-MSCs. A. Detections of the m6A levels of the total RNA in the HD-MSCs and AML-MSCs using EpiQuik m6A an RNA Methylation Quantification Kit; B. RT-qPCR detections of the METTL3 relative expressions in the HD-MSCs and AMLMSCs; C. Western Blot detections of the METTL3 relative expressions in the HD-MSCs and AML-MSCs 

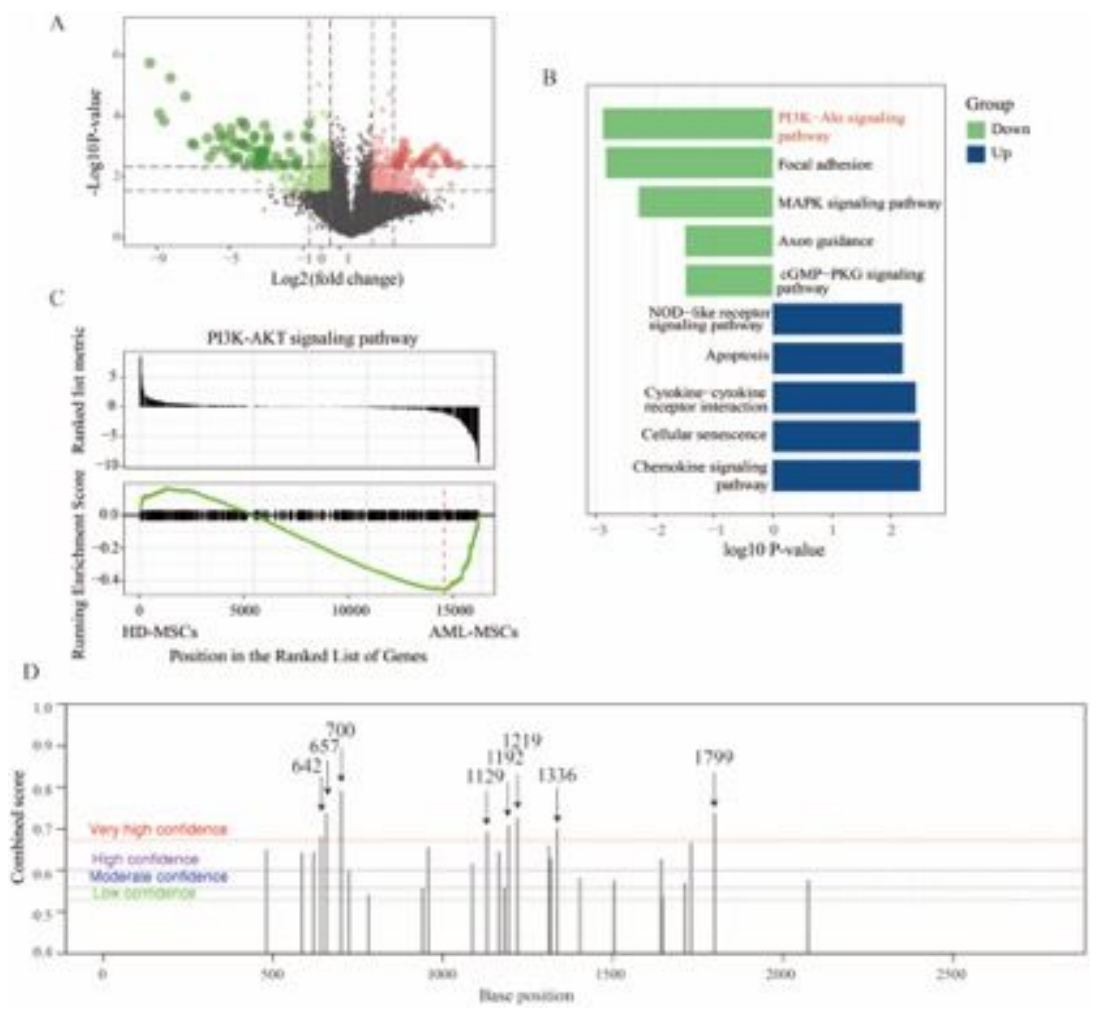

Figure 6

Significantly reduced m6A levels of AKT mRNA in the AML-MSCs. A. Volcanic map showing genes with different m6A levels in the AML-MSCs and HD-MSCs; B. KEGG analysis results showing different pathways with different m6A levels in the AML-MSCs and HD-MSCs; C. GSEA analysis results indicating the changes of the m6A levels in PI3K/AKT pathway genes in the AML-MSCs and HD-MSCs; D. Prediction results of the AKT gene full length predicted by SRAMP, in which the arrows and numbers in the figure represented the base positions corresponding to the AKT mRNA

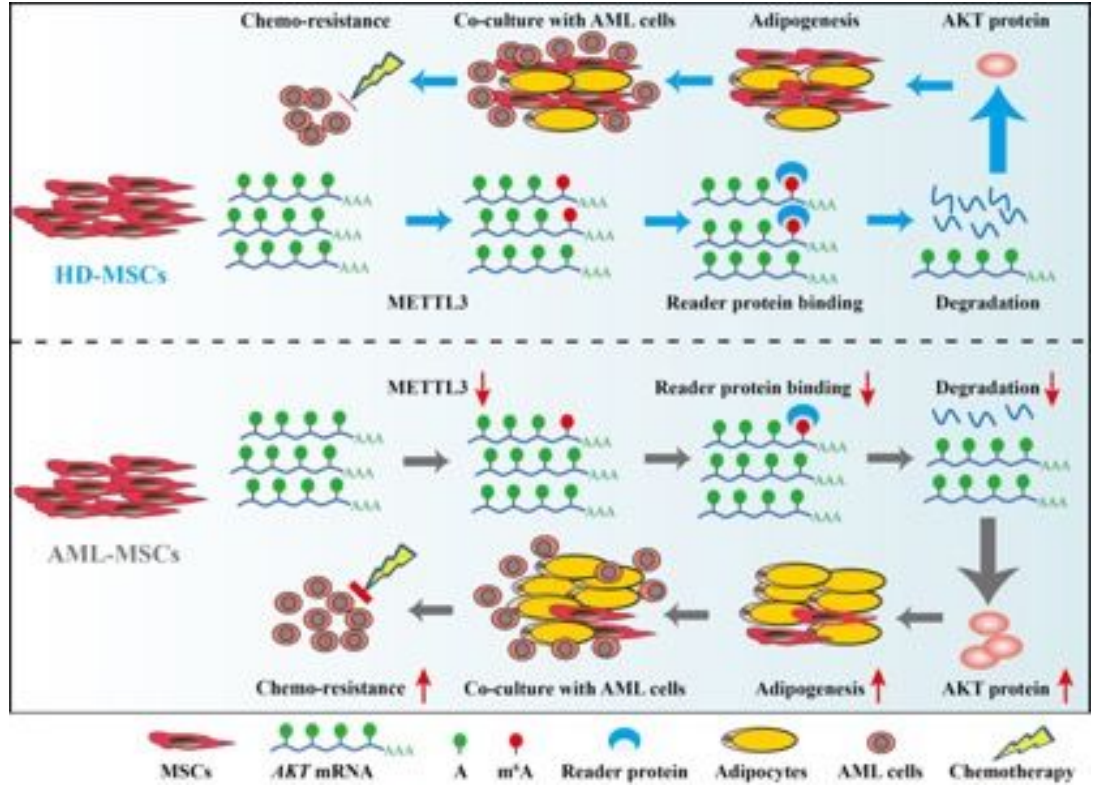

Figure 7 
Schematic image of the mechanism by which the METTL3 regulates the adipogenic differentiations of MSCs by targeting the PI3K/AKT signaling pathways affecting the chemo-resistance of the MSCs

\section{Supplementary Files}

This is a list of supplementary files associated with this preprint. Click to download.

- Supplementary.docx

- S1.png 